\title{
ASSOCIATION BETWEEN LOSS OF DP140 AND COGNITIVE IMPAIRMENT IN DUCHENNE AND BECKER DYSTROPHIES
}

\author{
Chamova $\mathrm{T}^{1, *}$, Guergueltcheva $\mathrm{V}^{1}$, Raycheva $\mathrm{M}^{1}$, Todorov $\mathrm{T}^{2,3}$, \\ Genova $\mathrm{J}^{2}$, Bichev $\mathrm{S}^{4}$, Bojinova $\mathrm{V}^{5}$, Mitev $\mathrm{V}^{3}$, Tournev $\mathrm{I}^{1,6}$, Todorova $\mathrm{A}^{2,3}$
}

"Corresponding Author: Teodora Chamova, M.D., Ph.D., Clinic of Neurology, University Hospital "Alexandrovska”, 1 Georgi Sofiiski str., Sofia 1431, Bulgaria; Tel.: +359-889802252; Fax: +359-2-9526787; E-mail: teodoratch@abv.bg

\begin{abstract}
The presence of variable degrees of non progressive cognitive impairment is recognized as a clinical feature of patients with Duchenne and Becker muscular dystrophies (DMD and BMD), but its pathogenesis still remains a matter of debate. A number of findings have proved that rearrangements located in the second part of the dystrophin (DMD) gene seem to be preferentially associated with cognitive impairment. Dp140 is a distal dystrophin isoform, mainly expressed during fetal brain development, whose role for neuropsychological functioning was suggested.

The aims of the current study were to explore the possible association between cognitive impairment and DNA mutations affecting the regulatory regions of Dp140, as well as to compare the neuropsychological functioning of patients affected with DMD and Intermediate muscular dystrophy (IMD) with those affected by Becker muscular dystrophy
\end{abstract}

\footnotetext{
${ }^{1}$ Clinic of Neurology, University Hospital "Alexandrovska," Sofia, Bulgaria

${ }^{2}$ Genetic Medico-Diagnostic Laboratory "Genica," Sofia, Bulgaria

${ }^{3}$ Department of Medical Chemistry and Biochemistry, Sofia Medical University, Sofia, Bulgaria

${ }^{4}$ National Genetics Laboratory, Molecular Medicine Center, Sofia Medical University, Sofia, Bulgaria

${ }^{5}$ Clinic of Child Neurology, University Hospital of Neurology and Psychiatry Sv. Naum, Sofia, Bulgaria

${ }^{6}$ Department of Cognitive Science and Psychology, New Bulgarian University, Sofia, Bulgaria
}

(BMD). Fiftythree patients genetically diagnosed with DMD, IMD and BMD, subdivided according to sites of mutations along the $D M D$ gene, underwent a neuropsychological assessment, evaluating their general cognitive abilities, verbal memory, attention and executive functions. Twenty patients with mutations, terminating in exon 44 or starting at exon 45 were tested by polymerase chain reaction (PCR) amplification of microsatellites STR44, SK12, SK21 and P20 DXS269, in order to evaluate the integrity of the Dp140 promoter region.

According to our statistical results, there was not a significant difference in terms of general intelligence between the allelic forms of the disease, a higher frequency of mental retardation was observed in DMD patients. The patients with BMD had better results on tests, measuring long-term verbal learning memory and executive functions. We found that patients lacking Dp140 performed more poorly on all neuropsychological tests compared to those with preserved Dp140. Overall, our findings suggest that the loss of Dp140 is associated with a higher risk of intellectual impairment among patients with dystrophinopathies and highlights the possible role of this distal isoform in normal cognitive development.

Keywords: Cognitive impairment; Duchenne muscular dystrophy (DMD); Becker muscular dystrophy (BMD) Dystrophin (DMD) gene; Intermediate muscular dystrophy (IMD); Dp140 isoform; Dystrophinopathies. 


\section{INTRODUCTION}

Duchenne/Becker muscular dystrophy (DMD/ BMD; OMIM\#310200; 300376) is an X-linked condition, caused by mutations in the enormously large $D M D$ gene, affecting approximately 1 in 3,500 live male births [1]. Clinically, DMD/BMD are characterized by progressive muscle wasting, loss of walking ability leading to complete wheelchair dependence, cardiomyopathy, and in some cases, by cognitive impairment. These two allelic forms differ in their severity, age of onset, and rate of progression.

The $D M D$ gene exhibits complex transcriptional regulation due to the presence of eight different promoters scattered throughout the gene, driving tissuespecific iso-forms of full-length dystrophin (Dp427M, Dp427-C, and Dp427-P) and several shorter isoforms. Two alternative full-length isoforms and three carboxy terminal proteins (Dp140, Dp116, Dp71), are expressed in the central nervous system (CNS) [2-8], probably related to neuropsycho-logical functions.

Cognitive impairment is a clinical feature of DMD, as it affects about one-third of the patients [9]. The DMD patient population was found to have an intelligence quotient (IQ) [shifted downward approximately one standard deviation (SD)] below the normal range [10]. Cognitive impairment is not progressive and does not correlate with the stage or the severity of the disease. It seems to affect verbal more than non verbal intelligence [11-13]. Males with BMD have a high incidence of learning difficulties. Autism, behavioral and attention problems are also more common in BMD than in the general population [14]. Deficits in verbal working memory and visual-motor integration have been identified $[15,16]$.

Neuropsychological impairment in patients with mutations in the $D M D$ gene is thought to be a consequence of complex interactions between different factors, such as deficiency or lack of dystrophin isoforms (Dp71; Dp140; Dp116) [12,16-20], with a subsequent reduction of regional brain glucose metabolism in areas rich in Dp, deficiency of dystrophin-like and dystrophin-associated proteins, mutations in genes closely located to the $D M D$ gene on the X-chromosome, accounting for developments such as XLRM, ACSL4, NXT2, GUCY2F, KCNE1L [21]. Some investigations have shown that mutations in the second part of the $D M D$ gene are more likely to be related to cognitive impairment $[10,12,17]$, by disturbing the regulation of the gene expression of the CNS dystrophin isoforms (Dp140, Dp71, Dp116) and subsequently, cause disruption of the neuronal maturation and development, the synapto-genesis and the intracellular homeostasis of the neurons and glial cells $[18,22,23]$. Dp140 is a brain distal isoform expressed during fetal development [19]. Its translation start site is located on exon 51 but its promoter and first exon lie in the large intron between exons 44 and 45 , a region of the $D M D$ gene that is commonly deleted in patients with dystrophinopathies. There have been several reports, showing a strong relation between loss of Dp140 and mental retardation in patients with dystrophinopathies $[10,12,15,24,25]$. We reviewed 53 patients genetically diagnosed with DMD, IMD and $\mathrm{BMD}$, and divided them into two groups, according to the expression of Dp140, with the aim of verifying a possible association between the loss of a Dp140 transcription unit and mental retardation in DMD, IMD and BMD patients.

\section{MATERIALS AND METHODS}

Fifty three patients were included in this study. The participating families were informed about the aim of the study and written informed consent was obtained from all patients and/or parents. They were diagnosed on the base of clinical symptoms, family history, EMG data, creatine kinase levels. The mean age of the patients was $15.87 \pm 14.13$ years, varying between 6 and 62. Their motor impairment was evaluated by the North star ambulatory assessment scale.

All participants underwent a formal cognitive assessment conducted by a neuropsychologist at the hospital site. None of them had a family history of neuropsychiatric disorders. The battery consisted of tests, evaluating general intelligence, verbal memory and executive functions. General intelligence was assessed by the Wechsler Intelligence Scale for children (HAWIK-R Bulgarian version, based on WISC$\mathrm{R})$ measuring full scale (FSIQ), verbal (VIQ), and performance IQ (PIQ). The verbal scale of WISC-R estimates verbal intelligence and it is based on completion of information, similarities, arithmetic, and vocabulary subtests. The performance scale of WISC$\mathrm{R}$ assesses non verbal intelligence and is computed from picture-completion, picture-arrangement, blockdesign, and object-assembly subtests. Verbal memory was assessed by the Rey auditory verbal learning test 
(RAVLT), consisting of a 15 -item list, that was presented five times, always in the same order, with an assessment of recall after each presentation as well as a second list (B) that was assessed only once. A recognition memory condition, consisting of 30 words was also included. The Tower of London (TOL) test was applied for the assessment of executive functioning, specifically to detect deficits in planning. The test consisted of two boards with pegs and several beads with different colors. The examiner used the beads and the boards to present the examinee with problemsolving tasks. Performances were considered either pathological or normal according to cut-off scores obtained by comparison with the existing norms.

All the participants were genetically confirmed either by MLPA (multiplex ligation-dependent probe amplification) or direct sequencing. The DNA samples were obtained from peripheral blood, using a DNA extraction kit (QIAamp DNA Mini Kit; Qiagen, Hilden, Germany), following the manufacturer's instructions. The MLPA analysis [SALSA MLPA P034/ P035 kit (www.mlpa.com)] was used as a first step to screen our patients for deletions/duplications along the $D M D$ gene. The analysis was performed according to the manufacturer's instructions [www.mlpa. com]. The obtained polymerase chain reaction (PCR) products were analyzed on an ABI PRISM ${ }^{\mathrm{TM}} 310$ genetic analyzer (Applied Biosystems, Foster City, CA, USA) in the presence of ROX500 size standard (Applied Biosystems). Each patient sample was analyzed simultaneously with at least two normal male samples. The MLPA data interpretation, in order to assess copy number changes (deletions) in comparison to the normal controls, was performed by the Excel program by Coffalyser MLPA data analysis software [www.mlpa.con]. The entire coding region of the $D M D$ gene, including exon/intron boundaries, was sequenced in patients with no detectable mutation on MLPA. The PCR products were purified by a PCR Product Pre-Sequencing Kit (Affymetrix Inc., Santa Clara, CA, USA), containing 4 U exonuclease $\mathrm{I}(10 \mathrm{U} / \mu \mathrm{L})$ and $0.8 \mathrm{U}$ shrimp alkaline phosphatase $(2 \mathrm{U} / \mu \mathrm{L})$. The sequencing reaction was performed by ABI PRISM ${ }^{\mathrm{TM}}$ BigDye Terminator v.3.1 Cycle Sequencing Kit (Applied Biosystems) and analyzed on an ABI PRISM ${ }^{\mathrm{TM}} 310$ genetic analyzer (Applied Biosystems). The sequencing profile was obtained by Sequencing Analysis v.3.4.1 software (Applied Biosystems). Twenty patients with mutations, terminating in exon 44 or starting at exon 45, were tested by PCR amplification of microsatellites STR44, SK12, SK21 and P20 DXS269, in order to evaluate the integrity of the Dp140 promoter region. The following single nucleotide polymorphism (SNPs) were chosen along intron 44 of the DMD gene: 5'-STR44 (CA)n GenBank M81257, IVS44SK12 (GT)n, IVS44SK21 (GT) n, P20 DXS269 (TC)n(TG)n GenBank M86524-3' [www.dmd.nl]. The PCR amplification was performed in $25 \mu \mathrm{L}$ total volume, containing $0.4 \mu \mathrm{M}$ primers, $0.2 \mathrm{mM}$ dNTPs, $1 \times$ supplied PCR reaction buffer (Genet Bio, Chungnam, Korea) and 0.5 U Prime Taq (Genet Bio). The annealing temperature was $58^{\circ} \mathrm{C}$. The obtained PCR products were analyzed on an ABI PRISM $^{\text {TM }} 310$ genetic analyzer (Applied Biosystems) in the presence of ROX500 size standard (Applied Biosystems) for 6-FAM-labeled primers or on ALFexpress (Pharmacia Biotech, Stockholm, Sweden) for Cy5-labeled primers.

Statistical analyses were conducted using statistical package IBM SPSS Statistics 19.0. A $p$ value below 0.05 was considered significant. The following statistical methods were used: descriptive statistics; student $T$-test for testing hypotheses for differences between two independent and two related samples; non parametric Mann-Whitney test for testing hypotheses for differences between two independent samples.

\section{RESULTS}

Genetic, clinical and neuropsychological data are summarized in Table 1. Of the 53 patients included in the study, $38(72.0 \%)$ were diagnosed as DMD, 13 $(24.0 \%)$ as BMD and two $(4.0 \%)$ were considered to have an intermediate phenotype. In total 30 (78.9\%) out of $38 \mathrm{DMD}$ patients were found to have molecular deletions in the $D M D$ gene, seven carried point mutations and one was found to have duplications in the $D M D$ gene. In the 38 DMD patients, the deletions with defined boundaries and the point mutations, were predicted to disrupt the dystrophin reading-frame. For the 13 BMD patients and two IMD patients, the identified deletions were inframe mutations. Deletions were heterogeneous in size and evenly distributed along the gene.

We compared the IQ of the patients with DMD, IMD and BMD, in order to verify if there is a statistically significant difference between the global intelligence of these allelic diseases, differing by the 
Table 1. Mutations and phenotype characteristics (motor impairment and intelligence quotient) of patients with dystrophinopathies.

\begin{tabular}{|c|c|c|c|c|c|c|c|}
\hline$\#$ & Mutation in the $D M D$ Gene & $\begin{array}{c}\text { Clinical } \\
\text { Phenotype }\end{array}$ & $\begin{array}{l}\text { Age at } \\
\text { Onset }\end{array}$ & $\begin{array}{c}\text { Age at } \\
\text { Assessment }\end{array}$ & \begin{tabular}{|c|}
$\begin{array}{c}\text { North Star Ambulatory } \\
\text { Assessment Scale }\end{array}$ \\
\end{tabular} & Dp140 & IQ \\
\hline 1 & c. $178 \mathrm{C}>\mathrm{T}, \mathrm{p} . \mathrm{G} \ln 60^{*}$, exon 3 & DMD & 3 & 9 & 16 & {$[+]$} & 113 \\
\hline 2 & c. $583 \mathrm{C}>\mathrm{T}, \mathrm{p} . \operatorname{Arg} 195^{*}$, exon 7 & DMD & 2 & 6 & 28 & {$[+]$} & 90 \\
\hline 3 & ex07ex39dup $\rightarrow$ c.531-?_5586+?dup & DMD & 5 & 9 & 30 & {$[+]$} & 102 \\
\hline 4 & ex08ex09del $\rightarrow$ c.650-?_960+_?del & DMD & 4 & 11 & 14 & {$[+]$} & 94 \\
\hline 5 & ex05ex15del $\rightarrow$ c.265-?_1812_?del & DMD & 3 & 8 & 2 & {$[+]$} & 71 \\
\hline 6 & c. $3220 \mathrm{G}>\mathrm{T}$, p.Glu1074*, exon 24 & DMD & 4 & 6 & 26 & {$[+]$} & 68 \\
\hline 7 & ex08ex42del $\rightarrow$ c.650-?_6117+?del & DMD & 1.5 & 10.5 & 1 & {$[+]$} & 100 \\
\hline 8 & ex12del $\rightarrow$ c.1332-?_1482+?del & DMD & 3 & 6 & 29 & {$[+]$} & 83 \\
\hline 9 & c.5704_5707delAGCC, exon 40 & DMD & 5 & 9 & 23 & {$[+]$} & 102 \\
\hline 10 & ex44del $\rightarrow$ c.6291-?_6438+?del & DMD & 4 & 9 & 32 & {$[+]$} & 124 \\
\hline 11 & ex44del $\rightarrow$ c.6291-?_6438+?del & DMD & 1.5 & 8 & 2 & {$[-]$} & 69 \\
\hline 12 & ex44del $\rightarrow$ c.6291-?_6438+?del & DMD & 3 & 9 & 1 & {$[-]$} & 68 \\
\hline 13 & ex44del $\rightarrow$ c.6291-?_6438+?del & DMD & 2 & 12 & 1 & {$[-]$} & 53 \\
\hline 14 & ex45del $\rightarrow$ c.6439-?_6614+?del & DMD & 2 & 8 & 26 & {$[-]$} & 74 \\
\hline 15 & ex45del $\rightarrow$ c.6439-?_6614+?del & DMD & 3 & 9 & 26 & {$[+]$} & 94 \\
\hline 16 & ex45ex49del $\rightarrow$ c.6439-?_7200+?del & DMD & 2 & 6 & 32 & {$[+]$} & 80 \\
\hline 17 & ex45ex50del $\rightarrow$ c.6439-?_7309+?del & DMD & 4 & 8 & 31 & {$[+]$} & 80 \\
\hline 18 & ex45ex54del $\rightarrow$ c.6439-?_8027_?del & DMD & 4 & 7 & 12 & {$[-]$} & 70 \\
\hline 19 & ex46ex49del $\rightarrow$ c.6615-?_7200+?del & DMD & 4 & 13 & 10 & {$[+]$} & 83 \\
\hline 20 & ex46ex50del $\rightarrow$ c.6615-?_7309+?del & DMD & 2 & 12.5 & 1 & {$[+]$} & 100 \\
\hline 21 & ex46ex50del $\rightarrow$ c.6615-?_7309+?del & DMD & 4 & 10 & 9 & {$[+]$} & 115 \\
\hline 22 & ex46ex52del $\rightarrow$ c.6615-?_7660+?del & DMD & 4 & 6 & 26 & {$[-]$} & 84 \\
\hline 23 & ex48ex50del $\rightarrow$ c.6913-?_7309+?del & DMD & 3 & 6 & 29 & {$[+]$} & 105 \\
\hline 24 & ex48ex52del $\rightarrow$ c.6913-?_7660+?del & DMD & 4 & 15 & 1 & {$[-]$} & 94 \\
\hline 25 & ex48ex52del $\rightarrow$ c.6913-?_7660+?del & DMD & 5 & 8 & 14 & {$[-]$} & 65 \\
\hline 26 & ex49ex51del $\rightarrow$ c.7099-?_7542+?del & DMD & 4 & 13 & 1 & {$[-]$} & 113 \\
\hline 27 & ex49ex52del $\rightarrow$ c.7099-?_7660+?del & DMD & 2 & 11 & 4 & {$[-]$} & 89 \\
\hline 28 & ex49ex52del $\rightarrow$ c.7099-?_7660+?del & DMD & 3 & 7.5 & 3 & {$[-]$} & 81 \\
\hline 29 & ex49ex52del $\rightarrow$ c.7099-?_7660+?del & DMD & 3 & 6 & 29 & {$[-]$} & 81 \\
\hline 30 & ex49ex54del $\rightarrow$ c.7099-?_8027+?del & DMD & 2 & 6 & 15 & {$[-]$} & 87 \\
\hline 31 & ex53del $\rightarrow$ c.7661-?_7872+?del & DMD & 3 & 6.5 & 20 & {$[+]$} & 89 \\
\hline 32 & ex53ex54del $\rightarrow$ c.7661-?_8027+?del & DMD & 3 & 16 & 0 & {$[+]$} & 74 \\
\hline 33 & ex53ex55del $\rightarrow$ c.7661-?_8217+?del & DMD & 4 & 15 & 0 & {$[+]$} & 65 \\
\hline 34 & c.8776C > T, p.Gln*, exon 59 & DMD & 4 & 8 & 24 & {$[+]$} & 92 \\
\hline 35 & c.8929_8932delAAAG, exon 59 & DMD & 4 & 7 & 26 & {$[+]$} & 94 \\
\hline 36 & ex61ex63del $\rightarrow$ c.9085-?_9286+?del & DMD & 3 & 13 & 2 & {$[+]$} & 86 \\
\hline 37 & ex79del $\rightarrow$ c.11047-?_(*2691_?)del & DMD & 6 & 15 & 0 & {$[+]$} & 90 \\
\hline 38 & c. $9361+\mathrm{G}>$ A, splice site & DMD & 2 & 6 & 29 & {$[+]$} & 68 \\
\hline 39 & ex03ex06del $\rightarrow$ c.94-?_530+?del & IMD & 6 & 16 & 21 & {$[+]$} & 88 \\
\hline 40 & ex03ex07del $\rightarrow$ c.94-?_649+?del & IMD & 6 & 9 & 27 & {$[+]$} & 89 \\
\hline 41 & ex03del $\rightarrow$ c.94-?_186+?del & BMD & 13 & 45 & 1 & {$[+]$} & 95 \\
\hline 42 & ex03ex07del $\rightarrow$ c.94-?_649+?del & BMD & 10 & 31 & 0 & {$[+]$} & 85 \\
\hline
\end{tabular}


Table 1. Continued

\begin{tabular}{|c|c|c|c|c|c|c|c|}
\hline \# & Mutation in the DMD Gene & $\begin{array}{c}\text { Clinical } \\
\text { Phenotype }\end{array}$ & $\begin{array}{r}\text { Age at } \\
\text { Onset }\end{array}$ & \begin{tabular}{c|} 
Age at \\
Assessment
\end{tabular} & $\begin{array}{c}\text { North Star Ambulatory } \\
\text { Assessment Scale }\end{array}$ & Dp140 & IQ \\
\hline 43 & ex45ex47del $\rightarrow$ c.6439-?_6912+?del & BMD & 20 & 30 & 32 & {$[+]$} & 85 \\
\hline 44 & ex45ex47del $\rightarrow$ c.6439-?_6912+?del & BMD & 15 & 28 & 30 & {$[+]$} & 90 \\
\hline 45 & ex45ex47del $\rightarrow$ c.6439-?_6912+?del & BMD & 17 & 57 & 1 & {$[+]$} & 105 \\
\hline 46 & ex45ex47del $\rightarrow$ c.6439-?_6912+?del & BMD & 12 & 29 & 32 & {$[-]$} & 90 \\
\hline 47 & ex45ex47del $\rightarrow$ c.6439-?_6912+?del & BMD & 10 & 36 & 14 & {$[+]$} & 75 \\
\hline 48 & ex45ex47del $\rightarrow$ c.6439-?_6912+?del & BMD & 23 & 62 & 1 & {$[+]$} & 90 \\
\hline 49 & ex45ex48del $\rightarrow$ c.6439-?_7098+?del & BMD & 28 & 46 & 28 & {$[+]$} & 80 \\
\hline 50 & ed45ex48del $\rightarrow$ c.6439-?_7098+?del & BMD & 19 & 46 & 26 & {$[+]$} & 95 \\
\hline 51 & ex45ex53del $\rightarrow$ c.6439-?_7872+?del & BMD & 29 & 41 & 13 & {$[-]$} & 75 \\
\hline 52 & ex45ex53del $\rightarrow$ c.6439-?_7872+?del & BMD & 9 & 9 & 33 & {$[-]$} & 65 \\
\hline 53 & ex45ex53del $\rightarrow$ c.6439-?_7872+?del & BMD & 0 & 7 & 34 & {$[-]$} & 83 \\
\hline
\end{tabular}

DMD: Duchenne muscular dystrophy; IMD: Intermediate muscular dystrophy; BMD: Becker muscular dystrophy.

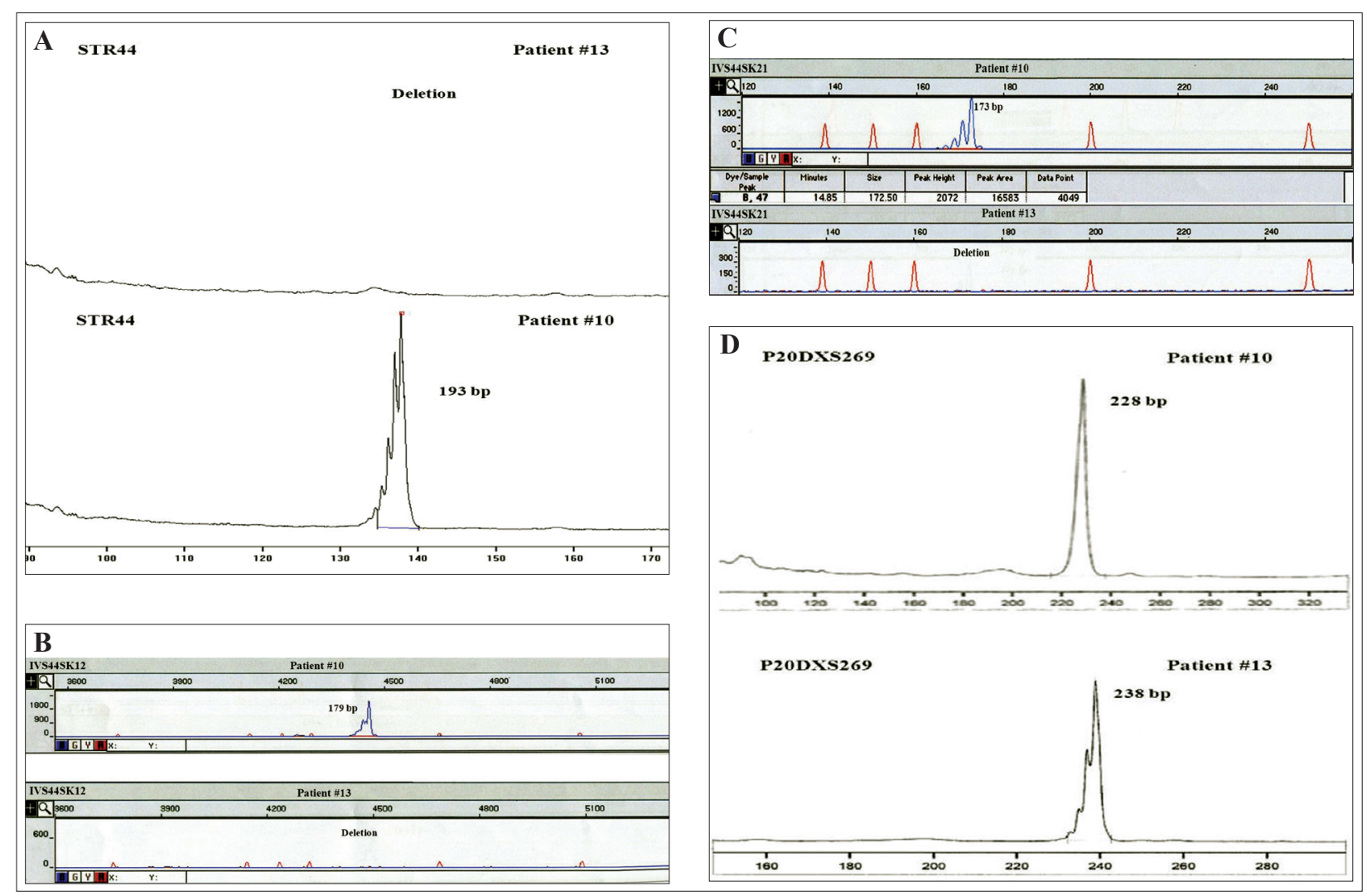

Figure 1. Results from amplification of microsatellites. A: STR44, B: SK12, C: SK21 and D: P20DXS269, a marker for the integrity of the Dp140 promoter region in patients \#10 and \#13. Patient \#10 is Dp140 positive and patient \#13 is Dp140 negative.

severity of muscle weakness. The mean IQ of our DMD and IMD patients was $86.98 \pm 15.34$, varying between 53 and 124, whereas the mean IQ of BMD patients was $85.62 \pm 10.40$, with a smaller variation between 65 and 105. Fifteen percent of the DMD and IMD patients $(6 / 40)$ had mild mental retardation, while $7.0 \%(1 / 13)$ of the BMD patients were mildly mentally retarded (IQ bellow 70). Four DMD 
Table 2. Comparative analysis of the neuropsychological test results of patients with Duchenne muscular dystrophy + Intermediate muscular dystrophy and Becker muscular dystrophy. (The statistically significant differences between the two groups $(\mathrm{p}<0.05)$ are in italics.)

\begin{tabular}{|c|c|c|c|c|c|}
\hline \multirow[t]{2}{*}{ Variable } & \multicolumn{2}{|c|}{ DMD + IMD } & \multicolumn{2}{|c|}{ BMD } & \multirow[b]{2}{*}{$p$ Value } \\
\hline & $n$ & Mean \pm SD & $n$ & Mean \pm SD & \\
\hline FSIQ & 40 & $86.98 \pm 15.34$ & 13 & $86.62 \pm 10.40$ & n.s.s. \\
\hline VIQ & 40 & $88.65 \pm 13.52$ & 13 & $79.50 \pm 14.85$ & - \\
\hline PIQ & 40 & $88.00 \pm 15.00$ & 13 & $74.50 \pm 7.78$ & - \\
\hline RAVLT trial $1 / 15$ & 40 & $4.73 \pm 1.30$ & 13 & $4.69 \pm 2.43$ & n.s.s. \\
\hline RAVLT trial 2/15 & 40 & $7.15 \pm 2.01$ & 13 & $7.85 \pm 3.31$ & n.s.s. \\
\hline RAVLT trial 3/15 & 40 & $8.48 \pm 1.93$ & 13 & $9.77 \pm 3.22$ & n.s.s. \\
\hline RAVLT trial 4/15 & 40 & $9.20 \pm 2.27$ & 13 & $11.00 \pm 2.97$ & 0.042 \\
\hline RAVLT trial 5/15 & 40 & $10.35 \pm 2.06$ & 13 & $11.62 \pm 2.79$ & 0.084 \\
\hline RAVLT immediate recall/75 & 40 & $40.08 \pm 7.57$ & 13 & $44.92 \pm 13.97$ & n.s.s. \\
\hline RAVLT list B/15 & 40 & $4.40 \pm 1.41$ & 13 & $4.85 \pm 1.99$ & n.s.s. \\
\hline RAVLT trial 6/15 & 40 & $8.78 \pm 2.60$ & 13 & $10.54 \pm 3.15$ & 0.049 \\
\hline RAVLT delayed recall & 40 & $8.53 \pm 2.24$ & 13 & $9.77 \pm 3.19$ & n.s.s. \\
\hline RAVLT recognition & 40 & $28.15 \pm 2.18$ & 13 & $28.08 \pm 2.10$ & n.s.s. \\
\hline TOL total move score & 40 & $39.25 \pm 19.38$ & 13 & $29.23 \pm 10.69$ & 0.046 \\
\hline TOL total correct score & 40 & $3.53 \pm 1.78$ & 13 & $4.23 \pm 1.88$ & n.s.s. \\
\hline TOL total rule violation & 40 & $2.73 \pm 3.24$ & 13 & $1.15 \pm 1.68$ & 0.083 \\
\hline TOL total time violation & 40 & $2.35 \pm 1.92$ & 13 & $0.85 \pm 1.21$ & 0.004 \\
\hline TOL initiation time & 40 & $58.65 \pm 21.44$ & 13 & $65.00 \pm 28.04$ & n.s.s. \\
\hline TOL-execution time & 40 & $386.15 \pm 174.47$ & 13 & $248.23 \pm 92.26$ & 0.002 \\
\hline TOL-total problem solving time & 40 & $445.70 \pm 179.57$ & 13 & $313.23 \pm 92.96$ & 0.008 \\
\hline
\end{tabular}

FISQ: full scale intelligence quotient; n.s.s.: not statistically significant; VIQ: verbal intelligence quotient;

PIQ: performance intelligence quotient; RAVLT: verbal learning test; TOL: Tower of London.

$(10.0 \%)$ and two BMD (16.0\%) patients showed a borderline cognitive level $(70<\mathrm{IQ}<79)$. Fourteen boys with DMD (35.0\%) and four BMD patients had low average intelligence $(80<\mathrm{IQ}<89)$, whereas 12 DMD and five BMD patients were considered to have normal intelligence. Four DMD patients $(10.0 \%)$ had high average or superior intelligence. There was not a statistically significant difference in IQ between the two allelic forms of the disease (Table 2). When comparing the results of the two groups, BMD patients had better results in some of the tests, related to long-term verbal learning memory, attention and executive functions (Table 2).

From the 20 patients with mutations terminating in exon 44 or starting at exon 45 , tested by PCR amplification of microsatellites STR44, SK12, SK21 and SK244, it was proved that seven had mutations impairing the promoter region of Dp140 (Figure 1). In two patients (\#52 and \#53), the deletion encompassed exon 51 as well. In another 10 patients, the expression of Dp140 was admitted to be impaired due to deletions affecting exon 51.

We compared neuropsychological data with genetic findings by dividing the group of patients with mutations in the $D M D$ gene in two populations according to the presence or absence of deletions in the Dp140 promoter and translation start site regions (Table 3). The mean age of the group with preserved Dp140 was $17.93 \pm 15.95$ and was $11.97 \pm 9.03$ for the group with impaired Dp140 expression. Our data 
Table 3. Comparative analysis of the neuropsychological test results between the Dp140 [-] and Dp104 [+] patients. (The statistically significant differences are in italics.)

\begin{tabular}{|c|c|c|c|c|c|}
\hline Variable & & $140-$ & & $140+$ & \\
\hline & $n$ & Mean \pm SD & $n$ & Mean \pm SD & $p$ Value \\
\hline FSIQ & 17 & $78.88 \pm 13.98$ & 36 & $90.31 \pm 12.95$ & 0.005 \\
\hline VIQ & 17 & $82.00 \pm 13.78$ & 36 & $91.67 \pm 12.33$ & 0.025 \\
\hline$P I Q$ & 17 & $80.27 \pm 13.17$ & 36 & $91.30 \pm 14.65$ & 0.200 \\
\hline RAVLT trial $1 / 15$ & 17 & $4.00 \pm 1.58$ & 36 & $5.06 \pm 1.55$ & 0.029 \\
\hline RAVLT trial 2/15 & 17 & $6.71 \pm 2.52$ & 36 & $7.61 \pm 2.28$ & 0.089 \\
\hline RAVLT trial 3/15 & 17 & $8.18 \pm 2.51$ & 36 & $9.08 \pm 2.25$ & n.s.s. \\
\hline RAVLT trial 4/15 & 17 & $9.06 \pm 2.54$ & 36 & $9.92 \pm 2.55$ & n.s.s. \\
\hline RAVLT trial 5/15 & 17 & $10.00 \pm 2.74$ & 36 & $10.97 \pm 2.02$ & n.s.s. \\
\hline RAVLT immediate recall/75 & 17 & $37.94 \pm 10.59$ & 36 & $42.83 \pm 8.41$ & n.s.s. \\
\hline RAVLT list B/15 & 17 & $4.18 \pm 1.81$ & 36 & $4.67 \pm 1.43$ & n.s.s. \\
\hline RAVLT trial $6 / 15$ & 17 & $8.06 \pm 2.59$ & 36 & $9.75 \pm 2.79$ & 0.040 \\
\hline RAVLT delayed recall & 17 & $8.00 \pm 2.76$ & 36 & $9.22 \pm 2.36$ & 0.068 \\
\hline RAVLT recognition & 17 & $26.65 \pm 2.71$ & 36 & $28.83 \pm 1.36$ & 0.004 \\
\hline TOL total move score & 17 & $49.06 \pm 23.47$ & 36 & $31.00 \pm 11.24$ & 0.007 \\
\hline TOL total correct score & 17 & $2.53 \pm 1.50$ & 36 & $4.25 \pm 1.70$ & 0.001 \\
\hline TOL total rule violation & 17 & $4.00 \pm 3.41$ & 36 & $1.56 \pm 2.47$ & 0.001 \\
\hline TOL total time violation & 17 & $3.06 \pm 2.59$ & 36 & $1.47 \pm 1.16$ & 0.044 \\
\hline TOL initiation time & 17 & $58.06 \pm 27.36$ & 36 & $61.22 \pm 21.15$ & 0.396 \\
\hline TOL execution time & 17 & $439.18 \pm 203.96$ & 36 & $311.43 \pm 133.26$ & 0.026 \\
\hline TOL total problem solving time & 17 & $497.41 \pm 209.99$ & 36 & $373.44 \pm 136.32$ & 0.040 \\
\hline
\end{tabular}

FISQ: full scale intelligence quotient; n.s.s.: not statistically significant; VIQ: verbal intelligence quotient; PIQ: performance intelligence quotient; RAVLT: verbal learning test; TOL: Tower of London.

showed a significant difference $(p<0.05)$ between general cognitive abilities in patients with an intact Dp140 isoform $(90.31 \pm 12.95) v s$. patients whose deletion was incompatible with formation of intact Dp140 (78.88 \pm 13.98$)$. According to the results from the Student $T$-test and Mann-Whitney test, the Dp140[-] population was found to perform more poorly on tests measuring verbal memory and executive functions compared to the $\mathrm{Dp} 140[+]$ population (Table 3 ).

\section{DISCUSSION}

Dystrophinopathies provide a unique opportunity to investigate cognitive impairment caused by mutations in a single gene and to analyze the impact of a dysfunctional dystrophin in the complex pathogenesis of cognitive impairment. Among patients with dystrophinopathies, there is a higher rate of cognitive disorders and mental retardation than in the normally developing population $[16,17]$. In the present study, we found that the patients with DMD and IMD had typically lower average intelligence with a mean IQ of $86.98 \pm 15.34$, which is $1 \mathrm{SD}$ below the population mean. The large SD reflects the enormous cognitive variation, found in the DMD patient group, whose IQs varied between 53 and 124 . The results are consistent with previous studies [22,24]. Our BMD group had an IQ of $85.62 \pm 10.40$, which is close to some previous reports [18]. According to our data, there was not a statistically significant difference between the aver- 
age IQ of patients with DMD, IMD and BMD on the one hand, but on the other hand, the percentage of mentally retarded DMD boys was greater (15.0 vs. $7.0 \%)$. The fact that in our study the number of DMD patients was larger than BMD patients shoud also be taken into consideration.

In recent years, our knowledge concerning the function of dystrophin in CNS has expanded. The etiology of cognitive disturbances of patients with dystrophinopathies have been widely investigated [9-12,16,17,20,22,23]. Over the past few years, short distal dystrophin isoforms (Dp140, Dp71) with major cerebral expression have been discovered. Although Dp140 function is still unclear, we found a significant difference in cognitive performance in patients with a presumably intact dystrophin isoform Dp140 vs. patients in whom this isoform was predicted to be absent in the brain, due to mutations in the regulatory region or translation start-site. Our data showed a significant difference in general cognitive abilities between patients with an intact Dp140 isoform ( 90.31 \pm 12.95 ) and patients whose deletion was predicted to be incompatible with the formation of an intact Dp140 (78.88 \pm 13.98$)$. Moreover, the Dp140[-] population was found to perform more poorly on tests measuring verbal memory, executive functions and attention compared to the Dp140[+] population.

These data support the increasingly recognized role of Dp140 in the cognitive functioning of the brain. The problem with cognitive impairment seems to be more complex. Although two of our patients were brothers, sharing the same defect, deletion of exons 45-53 of the DMD gene and the same social background, their IQ differed by 18 points ( 83 and 65). Thus, we can suppose there are some modifying factors that should be thoroughly investigated. This is in contrast to the findings of Taylor et al. [20], who described good concordance in IQ not only among siblings but also among unrelated patients with the same mutations.

In summary, the findings of the present study demonstrated that the cognitive profiles of patients with dystro-phinopathies is characterized by lower general intelligence compared to the healthy population. The frequency of mental retardation is higher in patients with DMD. Defective dystrophin isoforms (in particular Dp140) seem to be one of the major factors, influencing the neuropsychological development and functions in patients with DMD, IMD and BMD.

\section{ACKNOWLEDGMENTS}

The authors are grateful to the patients and their families for their collaboration. This study was supported by Grant DMU 02/21/2009: Investigation of genotype-phenotype correlations of cognitive impairment in patients with neuromuscular disorders was funded by the Ministry of Education and Science of Bulgaria.

\section{REFERENCES}

1. Griggs RC, Bushby K. Continued need for caution in the diagnosis of Duchenne muscular dystrophy. Neurology. 2005; 64(10): 1498-1499.

2. Barnea E, Zuk D, Simantov R, Nudel U, Yaffe D. Specificity of expression of the muscle and brain dystrophin gene promoters in muscle and brain cells. Neuron. 1990; 5(6): 881-888.

3. Boyce FM, Beggs AH, Feener C, Kunkel LM. Dys-trophin is transcribed in brain from a distant upstream promoter. Proc Natl Acad Sci USA. 1991; 88(4): 1276-1280.

4. Gorecki DC, Monaco AP, Derry JMJ, Walker AP, Barnard AE, Barnard PJ. Expression of four alternative dystrophin transcripts in brain regions regulated by different promoters. Hum Mol Genet.1992; 1(7): 505-510.

5. Hugnot JP, Gilgenkrantz H, Vincent N, Chafey $P$, Morris GE, Monaco AP, et al. Distal transcript of the dystrophin gene initiated from an alternative first exon and encoding a 75-kDa protein widely distributed in non muscle tissues. Proc Natl Acad Sci USA. 1992; 89(16): 7506-7510.

6. Lederfein D, Levy Z, Augier N, Mornet D, Morris $\mathrm{G}$, Fuchs $\mathrm{O}$, et al. A 71-kilodalton protein is a major product of the Duchenne muscular dystrophy gene in brain and other nonmuscle tissues. Proc Natl Acad Sci USA. 1992; 89(12): 5346-5350.

7. Rapaport D, Passos-Bueno MR, Takata RI, Campiotto S, Eggers S, Vainzof M, et al. A deletion including the brain promoter of the Duchenne muscular dystrophy gene is not associated with mental retardation. Neuromusc Disord. 1992; 2(2): 117-120.

8. Byers TJ, Lidov HGW, Kunkel LM. An alternative dystrophin transcript specific to peripheral nerve. Nat Genet. 1993; 4(1): 77-81. 
9. Pereira CC, Kiyomoto $\mathrm{BH}$, Cardoso $\mathrm{R}$, Oliveira AS. Duchenne muscular dystrophy, $\alpha$-dystroglycan immuno-expression in skeletal muscle and cognitive performance. Arq Neuropsiquiatr. 2005; 63(4): 984-989.

10. Felisari G, Martinelli Boneschi F, Bardoni A, Sironi M, Comi GP, et al. N. Loss of Dp140 dystrophin isoform and intellectual impairment in Duchenne dystrophy Neurology. 2000; 55(4): 559-564.

11. Billard C, Gillet P, Signoret JL, Uicaut E, Bertrand $\mathrm{P}$, Fardeau $\mathrm{M}$, et al. Cognitive functions in Duchenne muscular dystrophy: A reappraisal and comparison with spinal muscular atrophy. Neuromusc Disord. 1992; 2(5-6): 371-378.

12. Moizard M, Billard C, Toutain A, Berret F, Marmin N, Moraine C. Are Dp71 and Dp140 brain dystrophin isoforms related to cognitive impairment in Duchenne muscular dystrophy? Am J Med Genet. 1998; 80(1): 32-41.

13. Cotton S, Voudouris NJ, Greenwood KM. Intel-ligence and Duchenne muscular dystrophy: full-scale, verbal, and performance intelligence quotients. Dev Med Child Neurol. 2001; 43(7): 497-501.

14. Schneider SC, Tan G. Attention-deficit hyperactivity disorder. In pursuit of diagnostic accuracy. Postgrad Med. 1997; 101(4): 231-232.

15. Bardoni A, Sironi M, Felisari G, Comi GP, Bresolin N. Absence of brain Dp140 isoform and cognitive impairment in Becker muscular dystrophy. Lancet 1999; 353(9156): 897-898.

16. Young HK, Barton BA, Waisbren S, Dale LP, Ryan MM, Webster RI, et al. Cognitive and psychological profile of males with Becker muscular dystrophy. J Child Neurol. 2008; 23(2): 155-162.

17. D'Angelo MG, Bresolin N. Cognitive impairment in neuromuscular disorders. Muscle Nerve. 2006; 34(1): 16-33.
18. Bardoni A, Felisari G, Sironi M, Comi G, Lai M, Robotti M, et al. Loss of Dp140 regulatory sequences is associated with cognitive impairment in dystrophinopathies. Neuromusc Dis. 2000; 10(3): 194-199.

19. Lidov HGW, Selg S, Kunkel LM. Dp140: a novel $140 \mathrm{KDa}$ CNS transcript from the dystrophin locus. Hum Mol Genet. 1995; 4(3): 329-335.

20. Taylor PJ, Betts GA, Maroulis S, Gilissen C, Pedersen RL, Mowat DR, et al. Dystrophin gene mutation location and the risk of cognitive impairment in Duchenne muscular dystrophy. PLoS ONE 2010; 5(1): 1-9.

21. Bhat S, Schmidt K, Ladd S, Kim K, Schwartz $\mathrm{C}$, Simensen RJ, et al. Disruption of DMD and deletion of ACSL4 causing developmental delay, hypotonia, and multiple congenital anomalies. Cytogenet Genome Res. 2006; 112(1-2): 170-175.

22. Moizard M, Toutain A, Fournier D, Berret F, Raynaud M, Billard C, et al. Severe cognitive impairment in DMD: obvious clinical indication for Dp71 isoform point mutation screening. Eur J Hum Genet. 2000; 8(7): 552-556.

23. Anderson JL, Head SI, Rae C, Morely JW. Brain function in Duchenne muscular dystrophy. Brain. 2002; 125(Pt 1): 4-13.

24. Wingeier K, Giger E, Strozzi S, Kreis R, Joncourt F, Conrad B, et al. Neuropsychological impairments and the impact of dystrophin mutations on general cognitive functioning of patients with Duchenne muscular dystrophy. J Clin Neurosci. 2011; 18(1): 90-95.

25. D'Angelo MG, Lorusso, Civati F, Comi GP, Magri F, Del Bo R, et al. Neurocognitive profiles in Duchenne muscular dystrophy and gene mutation site. Pediatr Neurol. 2011; 45(5): 292-299. 